\section{Drs. Cao and Zheng reply}

\section{To the Editor:}

We appreciate Nagashima, $e t a l^{1}$ for the comments on our recent paper about a distinctive cutaneous feature in a subset of patients with classic dermatomyositis (DM) and clinically amyopathic $\mathrm{DM}^{2}$.

In our study, we found that patients with DM/clinically amyopathic dermatomyositis (CADM) who have ulcerative Gottron papules/Gottron sign with positive anti-MDA5 antibody and significantly lower baseline creatine kinase level are at increased risk of interstitial lung disease, especially acute/subacute interstitial pneumonia.

Nagashima, et al reported the survival of DM patients with interstitial lung disease (ILD) and ulcerated Gottron sign was not worse than that of patients without ulceration. The causes of inconsistent results may be attributed to the study population ${ }^{1}$. The study from Nagashima, et al's group focused on DM patients, excluding CADM. However, our cohort study consisted of patients with classic DM and CADM. Along with our data, the occurrence of ulcerative Gottron papules/Gottron sign was significantly higher in CADM than that in DM $(31.3 \% \text { vs } 11.1 \%, p=0.013)^{2}$. We reviewed the clinical course of the patients with ulcerative Gottron papules and Gottron sign, and the ulcers occurred after the presence of Gottron papules and Gottron sign or appeared simultaneously. In the patients reported by Nagashima, et al, 4 of the 15 patients had ulcers upon admission. We emphasized the ulcerations were evolved directly from Gottron papules and Gottron sign ${ }^{3}$. Hallmark clinical cutaneous manifestations of DM and CADM include Gottron papules and Gottron $\operatorname{sign}^{3}$. Nagashima, et al described the prognosis only of DM patients with Gottron sign with ulceration, whereas we included patients with not only Gottron papules but also Gottron sign with ulceration into analysis. The cumulative survival rate of the subsets with ulcerative Gottron papules/Gottron sign was significantly lower than the rate of those without ulceration $(\mathrm{p}<0.001)$.

Our study was consistent with Nagashima, et al's. Most of the patients having ulcerative Gottron papules and Gottron sign were treated with a high dose of glucocorticoids (1-2 mg/kg/day) combined with cyclophosphamide and/or intravenous immunoglobulin, but even then, $30.8 \%$ of patients died because of ILD and its complications (data were not shown). The prognosis of patients with ulcerative Gottron papules and Gottron rash will be further followed. More cases of CADM have been identified in other studies as clinicians have become increasingly aware of the condition ${ }^{4}$. It would be interesting and extremely valuable to be able to predict the onset of ILD and malignancy by distinctive rash.

HUA CAO, MD, PhD, Department of Dermatology, Ruijin Hospital, School of Medicine, Shanghai Jiao Tong University; JIE ZHENG, MD, PhD, Department of Dermatology, Ruijin Hospital, School of Medicine, Shanghai Jiao Tong University, Shanghai, China. Address correspondence to Dr. J. Zheng, Department of Dermatology, Ruijin Hospital, School of Medicine, Shanghai Jiao Tong University, No. 197 Ruijin Er Road, Shanghai 200025, China. E-mail: jie-zheng2001@126.com

\section{REFERENCES}

1. Nagashima T, Iwamoto M, Minota S. Gottron sign with ulceration is not a poor prognostic factor in patients with dermatomyositis and interstitial lung disease. J Rheumatol 2017;44:1099-1100.

2. Cao H, Xia Q, Pan M, Zhao X, Li X, Shi R, et al. Gottron papules and Gottron sign with ulceration: a distinctive cutaneous feature in a subset of patients with classic dermatomyositis and clinically amyopathic dermatomyositis. J Rheumatol 2016;43:1735-42.

3. Sontheimer RD. Cutaneous features of classic dermatomyositis and amyopathic dermatomyositis. Curr Opin Rheumatol 1999; 11:475-92.

4. Galimberti F, Li Y, Fernandez AP. Clinically amyopathic dermatomyositis: clinical features, response to medications and malignancy-associated risk factors in a specific tertiary-care-centre cohort. Br J Dermatol 2016;174:158-64.

J Rheumatol 2017;44:7; doi:10.3899/jrheum.170531 